\title{
General Electromagnetic Simulation Tool to Predict the Microwave Nonlinear Response of Planar, Arbitrarily-Shaped HTS Structures.
}

\author{
Josep Parrón, Carlos Collado, Jordi Mateu, Juan M. Rius, Nuria Duffo, Juan M. O'Callaghan.
}

\begin{abstract}
This work describes a simulation tool being developed at UPC to predict the microwave nonlinear behavior of planar superconducting structures with very few restrictions on the geometry of the planar layout. The software is intended to be applicable to most structures used in planar HTS circuits, including line, patch, and quasi-lumped microstrip resonators.

The tool combines Method of Moments (MoM) algorithms for general electromagnetic simulation with Harmonic Balance algorithms to take into account the nonlinearites in the HTS material. The Method of Moments code is based on a discretization of the Electric Field Integral Equation in Rao, Wilton and Glisson Basis Functions. The multilayer dyadic Green's function is used with Sommerfeld integral formulation. The Harmonic Balance algorithm has been adapted to this application where the nonlinearity is distributed and where compatibility with the MoM algorithm is required.

Tests of the algorithm in $\mathrm{TM}_{010}$ disk resonators agree with closed-form equations for both the fundamental and third-order intermodulation currents. Simulations of hairpin resonators show good qualitative agreement with previously published results, but it is found that a finer meshing would be necessary to get correct quantitative results. Possible improvements are suggested.
\end{abstract}

Index Terms - Nonlinar distortion, Method of Moments, Harmonic Balance, planar structures.

\section{INTRODUCTION}

Significant efforts are being made in the miniaturization of planar High Temperature Superconducting (HTS) filters to facilitate packaging and cooling, as well as to save substrate area [1]-[3]. These efforts are largely based on finding resonator shapes with small footprints, low resonant frequencies and high Qs. As a consequence, current densities in these resonators tend to be higher than those in standard resonator topologies. High current densities generate nonlinear effects in the HTS materials, which give rise to intermodulation products and degradation of the frequency response of the filters. Predicting these effects is complicated due to the difficulty of analyzing the nonlinear effects in the

Manuscript received September 18, 2000.

Authors are with Universitat Politènica de Catalunya (UPC), Campus Nord UPC D3, Barcelona 08034, Spain. (telephone 34-93-401 7229, email: joano@tsc.upc.es)

This work is supported by the Spanish Ministry of Education and Culture through project MAT-0984-C03-03 and scholarship AP99-78085980 for J. Mateu. J. Parrón is supported by the Generalitat de Catalunya, Comissionat per a Universitats i Recerca, under grant 1997 FI 00679 , convoluted shapes that miniature resonators have. We are currently working on a simulation tool that may help filter designers predict the nonlinear effects in a planar superconducting filter [4]. It should allow designers to vary the shapes of the resonators, to change the coupling among them, and to see how these changes affect the nonlinear properties of the filter. This tool combines routines for electromagnetic simulation of planar circuits based on the Method of Moments (MoM) [5] with Harmonic Balance (HB) routines that are normally used for the simulation of lumped-element circuits containing nonlinear components.

The Method of Moments calculates the surface current density on the planar metal (or superconducting) layout. In its standard (linear) version, the method can take into account the local surface impedance of the material $\left(Z_{s}\right)$ to relate the surface current density to the electric field. Similarly, the MoM-HB version that we propose allows for local nonlinear relations between surface current density and electric field. Furthermore, these relations need not be homogeneous throughout the HTS surface.

\section{LINEAR ANALYSIS}

A linear electromagnetic analysis of a planar circuit can be performed by relating the tangential components of incident and scattered electric fields to the surface current $\vec{J}_{s}(\vec{r})$ on the metals or superconductors in the circuit via [6]:

$\hat{n} \times\left(\vec{E}_{i}(\vec{r})+\left.\vec{E}_{s}(\vec{r})\right|_{s}=\hat{n} \times Z_{s} \vec{J}_{s}(\vec{r})\right.$

where $Z_{s}$ is the linear surface impedance of the materials.

The scattered fields generated by the induced current $\vec{J}_{s}(\vec{r})$ can be expressed in terms of vector and scalar potentials as:

$\vec{E}_{s}(\vec{r})=-j \omega \vec{A}(\vec{r})-\nabla \Phi(\vec{r})$

so the equation to solve is [6]:

$-\hat{n} \times\left.\vec{E}_{i}(\vec{r})\right|_{s}=\hat{n} \times\left(\left.(-j \omega \vec{A}(\vec{r})-\nabla \Phi(\vec{r}))\right|_{s}-Z_{s}(\vec{r}) \vec{J}_{s}(\vec{r})\right)$

The potentials $\bar{A}(\vec{r})$ and $\Phi(\vec{r})$ include information about the medium in which the planar layout is embedded (i.e, the effects of the various dielectric layers, ground plane, 
radiation, etc.) in their respective Green functions $\vec{G}_{A}\left(\vec{r} \mid \vec{r}^{\prime}\right)$ and $G_{\Phi}\left(\vec{r} \mid \vec{r}^{\prime}\right)$ :

$$
\begin{aligned}
& \vec{A}(\vec{r})=\int_{s} \ddot{G}_{A}\left(\vec{r} \mid \vec{r}^{\prime}\right) \vec{J}_{s}\left(\vec{r}^{\prime}\right) d s^{\prime} \\
& \Phi(\vec{r})=-\frac{1}{j \omega} \int_{s} G_{\Phi}\left(\vec{r} \mid \vec{r}^{\prime}\right) \nabla \cdot \vec{J}_{s}\left(\vec{r}^{\prime}\right) d s^{\prime}
\end{aligned}
$$

According to (3) and (4), the incident fields (those produced by the signal source in the absence of the metallic or superconducting layout) are related to the unknown induced surface currents through a linear operator $L_{E j}$ :

$-\hat{n} \times\left.\vec{E}_{i}(\vec{r})\right|_{s}=L_{E J}\left(\vec{J}_{s}(\vec{r})\right)$

As result ,we can express (5) in matrix form as:

$-\mathbf{E}_{1}=\mathbf{Z J}$

by applying the Method of Moments [5]. To do this, we decompose $\vec{J}_{s}(\vec{r})$ in Rao, Wilton and Glisson (RWG) linear triangle basis functions [7] and use the Galerkin Method with identical RWG testing functions [4].

To solve (6) we compute, store and invert the impedance matrix $\mathbf{Z}$. This is viable as long as the number of unknowns is small. If a larger number of unknowns were necessary to enhance the spatial resolution, we could use one of the different available iterative techniques, like the conjugate gradient (CG) or biconjugate gradient (BiCG), which combined with the multilevel matrix decomposition algorithm [8] have been shown to be particularly efficient in the analysis of planar structures in multilayered media [9]. This would allow us to obtain good results, with low computational requirements, in large problems.

\section{NONLINEAR ANALYSIS}

As detailed in [10], we assume that the electric field generated by a superconductor can be expressed in the time domain as a function of the current density and its derivates. This is a very general assumption, and includes most of the specific cases cited in the literature, like a penetration depth that depends on the current density, or a surface resistance that depends on the RF magnetic field [11],[12]. This dependence can be split in two terms, one gathering all the linear dependence, and a second including the nonlinearities:

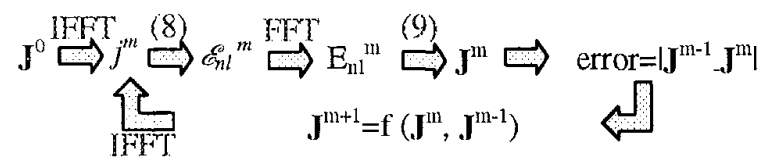

Fig. 1. Scheme for the MoM-HB algorithm. Uppercase variables: frequency domain, lowercase: time domain. Direct and inverse Fast Fourier Transform (FFT) are used to convert one to another.

$$
\overrightarrow{\mathscr{E}} \overrightarrow{\mathscr{E}}_{l}+\overrightarrow{\mathscr{E}}_{n l}^{a}=f_{t}\left(\vec{j}, \frac{\overrightarrow{d j}}{d t}, \ldots\right)+f_{n t}\left(\vec{j}, \frac{\overrightarrow{d j}}{d t}, \cdots\right)
$$

The linear contribution in (7) is considered through the surface impedance $Z_{s}$ in a standard MoM electromagnetic analysis that is performed previous to the consideration of the nonlinear effects in the material. In this paper, we will assume that the nonlinear term in (7) follows the form:

$$
\overrightarrow{\mathscr{E}}_{n l}=a_{n l}(\vec{j}) \cdot \vec{j}+\frac{d\left[b_{n l}(\vec{j}) \cdot \vec{j}\right]}{d t}
$$

to be consistent with the work in [10]. This is a simple form modeling a departure from linearity in the resistive and reactive parts of the surface impedance, and is also sufficiently general to be compatible with most published nonlinear HTS models [11],[12].Because of this non-linear behavior, when the device is excited with one or more harmonic signals, several new frequency components of the field may appear. All these can be understood as new contributions to the incident field at frequencies different form those of the signal source(s). So, in the frequency domain, each one of these $K+1$ components can contribute to the signal under analysis and has to satisfy the following equation:

$-\mathbf{E}_{i}^{k}+\mathbf{E}_{n l}^{k}=\mathbf{Z}^{k} \cdot \mathbf{J}^{k} \quad k=0 \ldots K$

To solve the nonlinear problem we have used the $\mathrm{HB}$ algorithm [13], which is an iterative method based on calculating the linear response using (9) in the frequency domain and the nonlinear one using (8) in the time domain. Transformations between domains are performed by direct and inverse FFT. The iterative process [4] begins by considering the linear solution $\mathbf{J}$ of (6) as the initial current vector. Fig. 1 shows the evolution of the process. Convergence is reached when the error is under a previously defined threshold.

\section{DISK RESONATOR}

In order to test the performance of the method of analysis proposed we consider a $\mathrm{TM}_{010}$ disk resonator on $\mathrm{LaAlO}_{3}$. There are two reasons that make this mode very adequate for this verification. The first is that there are no sharp variations in the current distribution, so the numerical results should not be very sensitive to the size of the mesh used for the MoM calculations. The second reason is that simple closed-form equations can be found to calculate the amplitude of the fundamental and spurious signals and compare these with the numerical results.

We excite a disk resonator with two fundamental tones $f_{l}$ and $f_{2}, 1 \mathrm{KHz}$ apart, to ensure that both are within the resonance band of the $\mathrm{TM}_{010}$ mode. It can be shown that the maximum value of current density for a disk resonator operating in the $\mathrm{TM}_{010}$ mode is 


$$
J_{f_{1}}^{\max }=1.445 \sqrt{P_{0} \frac{8 \beta}{1+2 \beta} Q_{L} \frac{1}{\omega_{0} \mu_{0} h \pi R^{2}}}
$$

where $P_{o}$ is the available power in the source, $\beta$ and $Q_{L}$ are, respectively, the coupling factor and the loaded quality factor obtained from the frequency response of the disk, $\omega_{0}$ ( $\left.=\omega_{1} \approx \omega_{2}\right)$ is the resonant frequency, $h$ the thickness of the dielectric and $R$ the disk radius.

At the same time, if we assume a quadratic dependence on the current density for the non-linear functions $a_{n l}(\vec{j})$ and $b_{n i}(\vec{j})$ of $(8)$, that is,

$a_{n l}(\vec{j}(\vec{r}))=\Delta r_{s} \cdot|\vec{j}(\vec{r})|^{2} ; \quad b_{n l}(\vec{j}(\vec{r}))=\Delta x_{s} \cdot|\vec{j}(r)|^{2}$

the current density maximum for the third order intermodulation product $2 f_{1}-f_{2}$ is [10]

$J_{2, f_{1}-f_{2}}^{\max }=1.11 \frac{\left|J_{f 1}^{\max }\right|^{2} J_{f_{2}}^{\max } Q_{L}}{\omega_{0} \mu_{0} h}\left[\Delta r_{s}+j \omega_{0} \Delta x_{s}\right]$

To find realistic values for $\Delta x_{s}$ we have followed the procedure outlined in [10] to derive an equation relating $\Delta x_{s}$ with the parameter $j_{I M D}$ used in [14]. The value of $\Delta x_{s}$ used in our simulations has been chosen to correspond to a typical value of $j_{I M D}$ of $10^{7} \mathrm{~A} / \mathrm{cm}^{2}$ [14]. We also choose $\Delta r_{s}=\omega_{0} \Delta x_{s}$ to set the value of $\Delta r_{s}$. According to (12), this makes the contribution of $\Delta r_{s}$ to the intermodulation current equal to that of $\Delta x_{s}$.

Fig. 3 shows the current density for the third order intermodulation product $2 f_{1}-f_{2}$. The radial distributions of the current density for one of the fundamental harmonics and for the third order intermodulation product $2 f_{1}-f_{2}$ are plotted in Fig. 4.

Comparing maximum values of current density in Fig. 4 with (10) and (12), we find a relative error below $5 \%$ in the

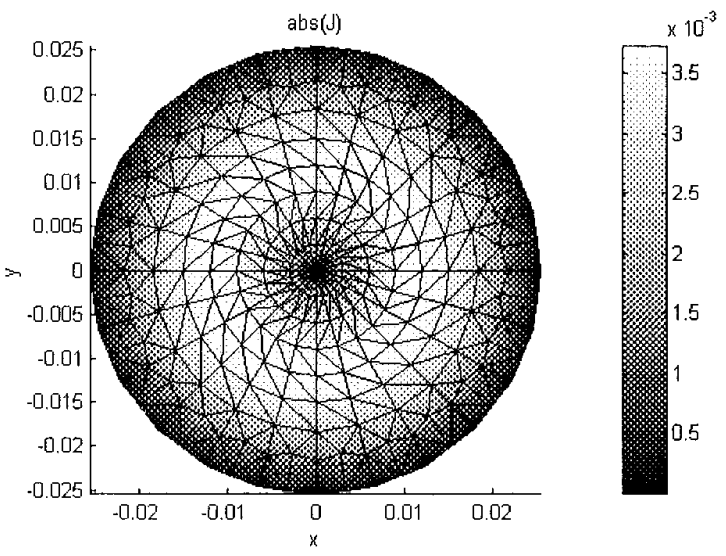

Fig. 3. Current density of the third-order intermodulation product in a $\mathrm{TM}_{010}$ disk resonator with a diameter of $50.8 \mathrm{~mm}$ and $\Delta \mathrm{r}_{s}=2.810^{-12}$ $\left(\Omega \cdot \mathrm{m}^{2}\right) / \mathrm{A}^{2}, \Delta \mathrm{x}_{\mathrm{s}}=310^{-22}\left(\mathrm{H} \cdot \mathrm{m}^{2}\right) / \mathrm{A}^{2}$.

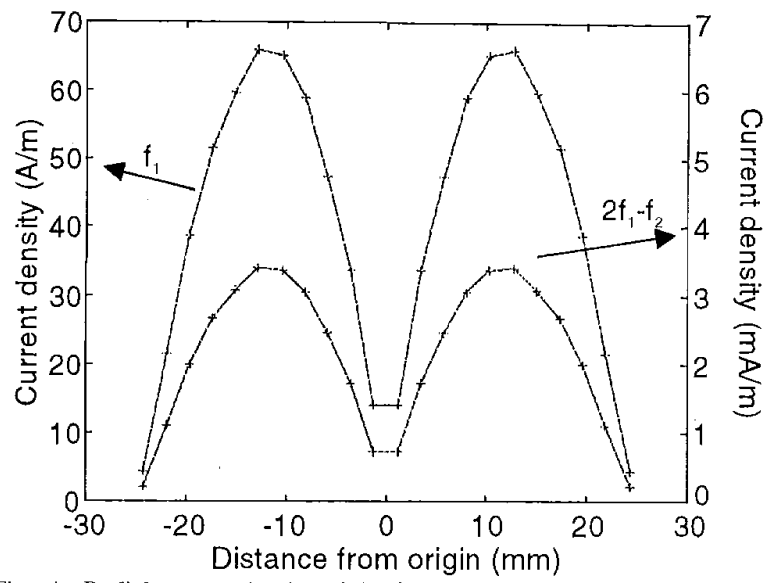

Fig. 4. Radial current density of the fundamental and third-order spurious obtained with MoM-HB. Deviations from theoretical values are smaller than 5 and $10 \%$ respectively.

fundamental tone and $10 \%$ for the intermodulation product. There are no significant changes in the numerical results when the size of the mesh is reduced beyond that which corresponds to 700 meshing triangles in the whole disk. This suggests that the meshing size is adequate to analyze this distributed nonlinear problem with a reasonable computational burden. A single-frequency simulation with about 700 meshing triangle on a Pentium II PC running at $550 \mathrm{MHz}$ takes about 50 seconds.

\section{HAIRPIN RESONATOR}

We have analyzed hairpin resonators to test the performance of our software when simulating different resonant modes in a given structure. In the test configuration described in [14], the even and odd resonant modes in these resonators show very different behavior.

Fig. 5 shows the resonator layouts and the intermodulation current density for the value of $\Delta x_{s}$ used in the previous section (in this section, $\Delta r_{s}$ is made zero to be consistent with the model used in [14]).

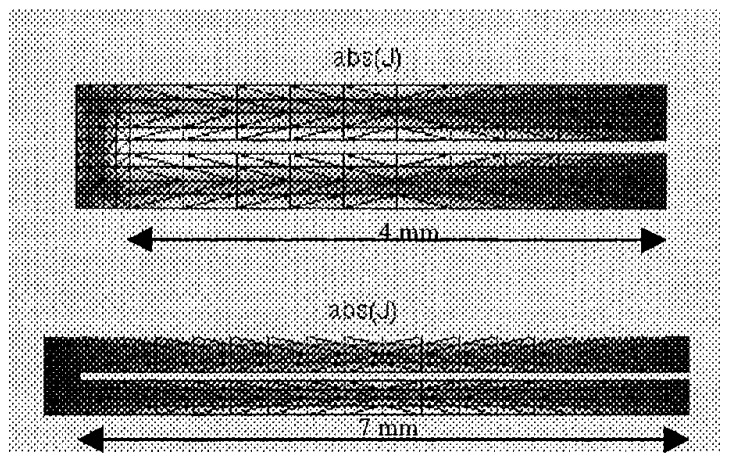

Fig. 5. Current density of the third-order intermodulation product in a microstrip hairpin resonator with $\Delta \mathrm{r}_{5}=0, \Delta \mathrm{x}_{5}=310^{-22}\left(\mathrm{H} \cdot \mathrm{m}^{2}\right) / \mathrm{A}^{2}$. The layout and other experimental features correspond to those used in [14]. The through line is omitted in this figure to enhance the detail of the current density distributions. Note that current maxima are placed at the inner edges in the smaller resonator $(l=\lambda / 2)$, and on the outer edges in the resonator with $1 \approx \lambda$. 


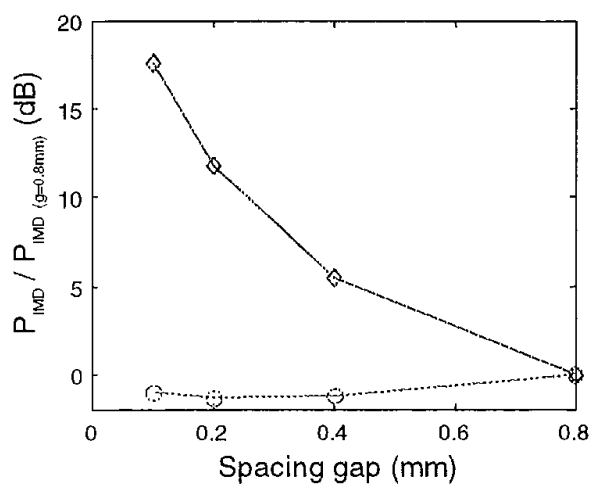

Fig. 6. Simulated intermodulation power produced by two hairpin resonators as a function of the gap between the two hairpin lines. Results are normalized with respect to the power produced when the gap is $800 \mu \mathrm{m}$ wide. Data marked with circles corresponds to a resonator with $1 \approx \lambda$ (even mode); diamonds correspond to a resonator with $l=\lambda / 2$ (odd mode).

Fig. 6 shows the normalized values of intermodulation power at the load $\left(P_{I M D}\right)$ of several simulations varying the spacing $(g)$ between the arms of the resonator. This is made for the first odd $(l \approx \lambda / 2)$ and the first even $(l \approx \lambda)$ resonant modes. As found in [14], there is not a significant dependence of the intermodulation power on the gap spacing for the even mode, whereas a much stronger dependence is found in the odd mode. According to Fig. 6, the normalized value of $P_{I M D}$ for the odd mode increases as the spacing $g$ is reduced. This indicates that the nonlinear effects in this mode are stronger than those in the even modes, in agreement with the experimental results in [14]. This is due to the different field configurations in the modes, which makes current densities in the odd modes much higher than those in the even modes. These maxima occur at the inner edges of the hairpin structure in even modes, and at the outer edges in odd modes. These features are reproduced in the current distributions shown in Fig. 5.

Even though the qualitative results are correct, a finer meshing is still needed to obtain good quantitative data. The intermodulation power $P_{I M D}$ changes by a factor of five when the meshing size is increased by a factor of two. We have not gone beyond using eight cells in a cross section of a $400 \mu \mathrm{m}$ wide microstrip line, and we do not expect to get accurate results unless our mesh size around the current maxima is on the order of the penetration depth. Our future work will be geared towards adequate meshing strategies permitting an efficient operation of this software on a PC computer.

\section{CONCLUSIONS}

We have tested our current version of MoM-HB software in several types of planar microwave resonators.

One of the test structures is a disk resonator, for which one can derive closed-form equations that relate the material parameters with the intermodulation power produced by the resonator. We have found good agreement between the simulations and the theoretical results. Also, the current distribution is smooth and this facilitates the meshing of the structure for the MoM algorithm. We have not found a strong dependence of our simulations with the meshing size, provided that this is sufficiently fine.

Two hairpin resonators have also been simulated: one resonating in an even mode, and another in an odd mode. The qualitative trends in [14] (a much stronger nonlinear behavior of the odd mode resonator) have been reproduced. However, the current in these resonators has sharp peaks at the edges of the microstrip lines, which make meshing difficult. Our quantitative results still depend too strongly on the meshing size, and we are looking for ways to solve this. Reducing the meshing size increases the computational burden, and we try to keep this low enough to be done by a PC computer. Possible solutions might be found by using the methods mentioned in Sect. II .

\section{ACKNOWLEDGMENT}

The authors want to acknowledge Salvador Talisa for helpful comments and suggestions.

\section{REFERENCES}

[1] M.J. Lancaster, F. Huang, A. Porch, B. Avenhaus, J-S. Hong and D.Hung, "Miniature superconducting filters", IEEE Trans on Microwaves Theory and Techniques, Vol. 44, No 7, July 1996.

[2] J-S. Hong and M.J. Lancaster, " Couplings of microstrip square openloop resonators for cross-coupled planar microwave filters", IEEE Trans on Microwaves Theory and Techniques, Vol. 44, No 12, July 1996.

[3] K. Huang, D. Hyland, A. Jenkins, D. Edwards and D. Dew-Hughes, "A miniaturized interdigital microstrip bandpas filter", IEEE Trans on Microwaves Theory and Techniques, Vol. 9, No 2, July 1999.

[4] J. Parrón, C. Collado, J. Mateu, E. Ubeda, J.M. Rjus, J.M. $O^{\prime}$ Callaghan, "Calculation of RF spurious in planar superconducting structures", Inst. of Phys. Conf. Ser. No. 167, pp 339-342, 2000.

[5] R. F. Harrington, Field Computation by Moment Methods (New York: MacMillan), 1968.

[6] J. R. Mosig Numerical Techniques for Microwave and MillimeterWave and Passive Structures, Itoh T, (New York: John Wiley \& Sons) pp 133-213, 1989

[7] S. M. Rao, D. R. Wilton, A. W. Glisson, "Electromagnetic scattering by surfaces of arbitrary shape", IEEE Trans. Antennas and Propag., Vol. 30, No 3, pp. 409-18, 1982.

[8] J. M. Rius, E. Úbeda, J. Parrón, J. R. Mosig, "Multilevel matrix decomposition algorithm for analysis of electrically large electromagnetic problems in 3-D", Microwave and Optical Technology Letters, Vol 22, No 3, 1999

[9] J.M. Rius, J. Parrón, E. Úbeda, J.R. Mosig, "Analysis of microstrip antennas by multilevel matrix decomposition algorithm", Millenium Conference on Antennas and Propagation (AP2000), Davos, Switzerland, 9-14 April 2000

[10] J.Mateu, C. Collado and J.M O'Callaghan," Nonlinear analisis of disk resonators. Application to material characterization and filter design". Submitted to IEEE Transactions on Applied Superconductivity (ASC2000 issue)

[11] B.A. Willemsen, T. Dahm, B.H. King and D.J. Scalapino," Microwave intermodulation in high-Tc superconducting microstrip resonator", IEEE Transactions on Applied Superconductivity, Vol. 9, June 1999.

[12] P.P Nguyen, D.E. Oates, G. Dresselhaus, M.S. Dresselhaus, "Nonlinear surface impedance for $\mathrm{Yba}_{2} \mathrm{Cu}_{3} \mathrm{O}_{7-\mathrm{x}}$ thin films: Measurements and a coupled-grain model", Physical Review B, Vol. 48, No. 9, 1993

[13] S. A. Maas, Nonlinear Microwave Circuits , Artech House, 1988.

[14] B. A. Willemsen, T. Dahm and D. J. Scalapino, "Microwave intermodulation in thin film high-Tc superconducting microstrip hairpin resonators: Experiment and theory", Appl. Phys. Lett. Vol. 71, No. 29, pp. 3898-3900, 1997. 